\title{
Enhanced DNA Separation Rates in Nano-Platinum Doped Agarose
}

\author{
Shantanu Bhattacharya ${ }^{1,4,7}$, Nripen Chanda ${ }^{3}$, Yi-Shao Liu ${ }^{4}$, Sheila A. Grant ${ }^{1}$, \\ Keshab Gangopadhyay ${ }^{2,6}$, Paul R. Sharp ${ }^{3}$, Rashid Bashir ${ }^{4,5, *}$, and Shubhra Gangopadhyay²,* \\ ${ }^{1}$ Department of Biological Engineering, University of Missouri, Columbia, MO, 65211, USA \\ ${ }^{2}$ Department of Electrical and Computer Engineering, University of Missouri, Columbia, MO, 65211, USA \\ ${ }^{3}$ Department of Chemistry, University of Missouri, Columbia, MO, 65211, USA \\ ${ }^{4}$ Birck Nanotechnology Center, Department of Electrical and Computer Engineering, \\ Weldon School of Biomedical Engineering, Purdue University, Purdue University, West Lafayette, IN, USA \\ ${ }^{5}$ Micro and Nanotechnology Laboratory, 2000 MC-249, University of Illinois at Urbana-Champaign, \\ Urbana, Illinois 61801, IL, USA \\ ${ }^{6}$ Nuclear Science and Engineering Institute, University of Missouri, Columbia, MO, USA \\ ${ }^{7}$ Department of Mechanical Engineering, Indian Insitute of Technology, Kanpur, UP, India
}

\begin{abstract}
In this work, nanoparticle-doped matrices for DNA separation at low voltages are described. High conductivity agarose gels doped with platinum nano-particles have been synthesized and characterized by TEM, EDS and SEM. This new doping technique for agarose gels enhances the dielectric constant of the gels by up to 1.5 folds as compared to undoped gel, decreases the resistance (from 97 ohms to about 60 ohms) and increases DNA mobility by 1.5 times (from $6.6 \times 10^{-5} \mathrm{~cm}^{2} / \mathrm{V} \cdot \mathrm{sec}$ to $\left.9.3 \times 10^{-5} \mathrm{~cm}^{2} / \mathrm{V} \cdot \mathrm{sec}\right)$ at lower operating electric fields $(8 \mathrm{~V} / \mathrm{cm})$. We believe that the faster movement of DNA arises from an increased dielectric constant and a reduced resistance of the doped material resulting in increased ionic mobility. Using image analysis tools, we have also observed that there is no band broadening effects in the platinum doped gel sample, which indicate no appreciable temperature rise due to incorporation of platinum nanoparticles in agarose gel.
\end{abstract}

Keywords: Dielectric, DNA, Electrophoresis, Mobility, Nanotechnology.

\section{INTRODUCTION}

DNA electrophoresis is a critical but time consuming step in many genetic analyses. In order to address this issue, several technologies to enable DNA electrophoresis with separation at high speeds have been realized over the last two decades. ${ }^{1}$ Miniaturized integrated gene analysis systems are especially promising towards these goals. $^{2-4}$ These systems demonstrate an overall reduction in size, reduced use of reagents, rapid and accurate analysis, and increased portability for field usage. The field applications for such devices, however, are limited by the voltage requirements that can be as high as tens of kilovolts $(10-30 \mathrm{kV})$ due to the highly resistive capillary columns. ${ }^{5}$ The development of DNA separation matrices for faster and higher resolution DNA separation remains an important research endeavor. The ability to size fractionate nucleic acids through capillaries at lower voltages is highly desirable. ${ }^{6,7}$ Earlier work

${ }^{*}$ Authors to whom correspondence should be addressed. has demonstrated capillary electrophoresis in a separation channel partitioned into smaller separation zones using closely spaced electrode pairs; ${ }^{8}$ however, this technique uses very complicated device architecture and associated controls. Till date no exploratory research has indicated the use of conducting separation matrices with enhanced dielectric constant for size fractionation of DNA which can be directly applied to micro-channels in performing capillary electrophoresis.

It is well established that metal nano-particles can significantly alter electrical properties of various polymers and other materials. Lu et al. ${ }^{9}$ has used surface modified silver nanoparticles to formulate nanocomposites with epoxy matrices. The modified nanocomposites demonstrate high dielectric constants elevated by around 5 times. Xu et al. ${ }^{10}$ have demonstrated composites made of Aluminum nanoparticles and different polymer matrices like epoxy, silicone, Polyimide, Polycarbonate, Benzocyclobutene (BCB) etc. with high dielectric constant and lower dissipation factor. In another instance, the surface modification of $\mathrm{TiO}_{2}$ nanoparticles by a polar silane 
coupling agent could effectively improve both the dielectric breakdown strength and space charge distribution of polyethylene composites. ${ }^{11,12}$ Muszynska et al. ${ }^{13}$ have demonstrated an overall increase in conductivity of a polyethylene oxide matrix using $\mathrm{Al}$ filler nanoparticles in concentration range $0.25-5 \mathrm{wt} \%$. Beyond this miniscule range a decrease in conductivity is observed. Oh et al. ${ }^{14}$ have demonstrated an increase in conductivity of Polyethylene oxide (PEO) which cross-links with Polyethylene glycol dimethacrylate (PEGDMA) to form a gelled polymer electrolyte, using lithium salts. Here the ionic conductivity is found to enhance with an increase in the lithium content. Morita et al. ${ }^{15}$ have demonstrated an increase in the conductivity of the polymeric matrix comprised of PEO-PMA and $\mathrm{Li}\left(\mathrm{CF}_{3} \mathrm{SO}_{2}\right)_{2} \mathrm{~N}$ by addition of ceramic filler materials like $\alpha$ and $\gamma \mathrm{Al}_{2} \mathrm{O}_{3}$ respectively. This increase in conductivity is due to an increase in the highly conductive amorphous phase on the grain matrix border. With an increase in the filler concentrations the thickness of this shell along the grain boundaries increases leading to an easy flow path for current which is concentrated in the percolation paths formed by the grain shells. ${ }^{16}$ In this work, we have developed a protocol for doping agarose gels with externally synthesized platinum nano-particles. ${ }^{17}$ The platinum particles used in this work were chemically synthesized using a standard reduction process and are doped into agarose gel. Because of this doping process the dielectric capacitance of the composite was found to increase, as determined by the use of impedance spectroscopy. The doped agarose films showed an overall increase in mobility of the DNA. The gel material was also incorporated in thin PDMS capillaries and capillary electrophoresis was demonstrated at a lower voltage. The increase in mobility and the higher conductivity was attributed to the enhanced dielectric constant of the agarose platinum composite. A check of the resolution of the new gel material with 100-1000 bp gene marker indicated a negligible change in resolution. The new gel material does not lead to any band broadening effects which may happen if the temperature of the gel is increased due to increased conductivity. ${ }^{18}$ We used imageJ software to analyze the differences in the band area to study the broadening effects and found identically sized bands on both gels using a $1 \mathrm{Kbp}$ ladder. Capillary electrophoresis experiments performed inside PDMS channels using this new material, reported separately, ${ }^{19}$ indicated a stain movement within capillaries at a reduced voltage of $60 \mathrm{~V}$. [Refer to Fig. S4 in supporting information].

\section{EXPERIMENTAL DETAILS}

Platinum nano-particle hydrosols at two different concentrations were prepared by reducing an aqueous solution of potassium platinum (II) tetrachloride $\left(\mathrm{K}_{2} \mathrm{PtCl}_{4}, 5.8 \mathrm{mM}\right.$ and $11.6 \mathrm{mM})$ with sodium borohydride $(137.2 \mathrm{mM}$ and $274.4 \mathrm{mM}$ ) in the presence of mercapto succinic acid
(MSA, $26.7 \mathrm{mM}$ and $53.4 \mathrm{mM}$ ) in a Schlenk flask under an argon atmosphere. For both concentrations, the molar ratio of MSA and $\mathrm{K}_{2} \mathrm{PtCl}_{4}$ was $0.46(\mathrm{~S} / \mathrm{Pt})$. The formation of the platinum hydrosol was monitored by observing a change in coloration. ${ }^{17}$ Both hydrosols were investigated with a Shimadzu UV-Vis spectrophotometer to confirm the presence and sizes of platinum nano-particles (sharp peak at $220 \mathrm{~nm}) .{ }^{20}$ A JEOL $1200 \mathrm{EX}$ transmission electron microscope (TEM) was used to further characterize the size of the particles. The mean particle size and size distribution were obtained from the digitized photo images using Adobe Photoshop software. Each platinum hydrosol solution was separately mixed with $1 \mathrm{X}$ TAE (Triacetate buffer, $\mathrm{pH}=8.4, \mathrm{M} / \mathrm{S}$ Fisher BioReagents) buffer sample in 1:4 volume ratio. Molecular biology grade low EEO (electroendosmotic flow) agarose was mixed with this solution to give a $2 \%$ (weight/vol) gel after heating the mixture to $80{ }^{\circ} \mathrm{C}$ followed by cooling to room temperature.

Slab gel electrophoresis was conducted with and without platinum hydrosol wherein a 527 bp amplified [PCR based] viral DNA sample was driven through these gels at identical voltages. A digital image was acquired using a Kodak inverted camera after 10, 15 and 20 min intervals at voltages ranging from 50-200 V and a comparison of DNA mobility in both gel materials was performed (see Fig. 1).

The gel dielectric constants and resistance were measured using impedance spectroscopy techniques wherein a set of interdigitated micro-fabricated Pt electrodes over a silicon wafer are used to determine the impedance of the medium ${ }^{21}$ (see Fig. 2(a)). The electrodes were connected to two bond pads over which micro-manipulator probe tips were used to measure the impedance over a frequency range of $100 \mathrm{~Hz}-100 \mathrm{kHz}$ using an Agilent 4284A precision LCR meter using a Lab View interface.

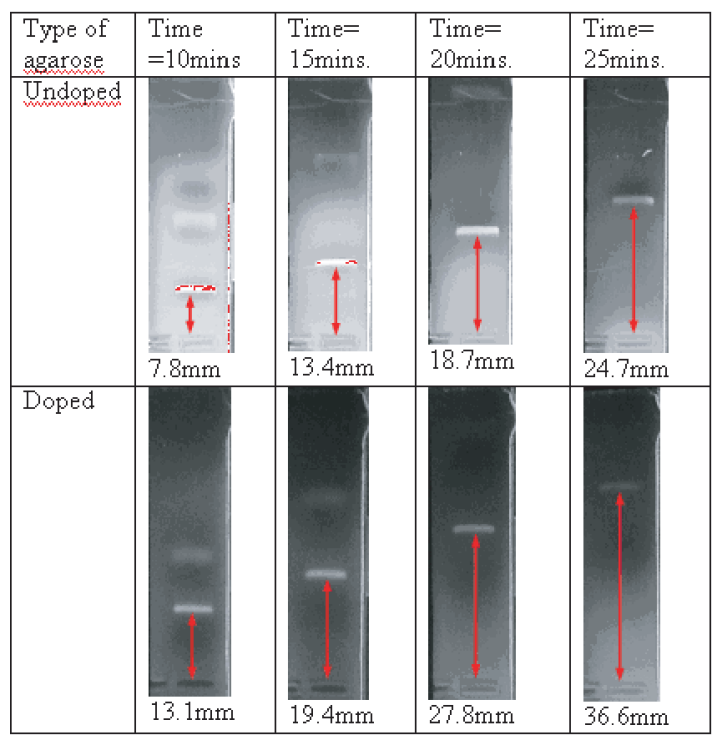

Fig. 1. Images of the fluorescent band in plain and platinum doped agarose gel taken at different times for $200 \mathrm{~V}$ applied voltage. 


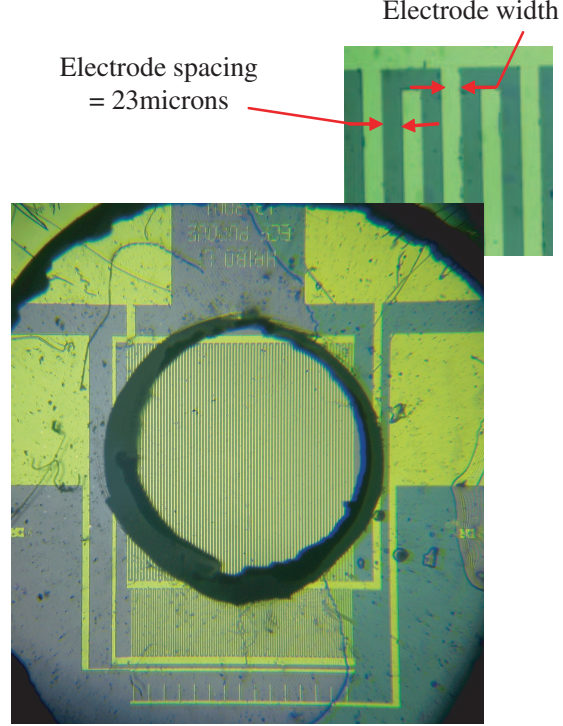

(a)

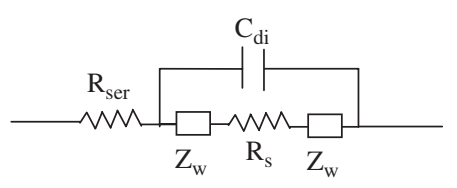

(b)

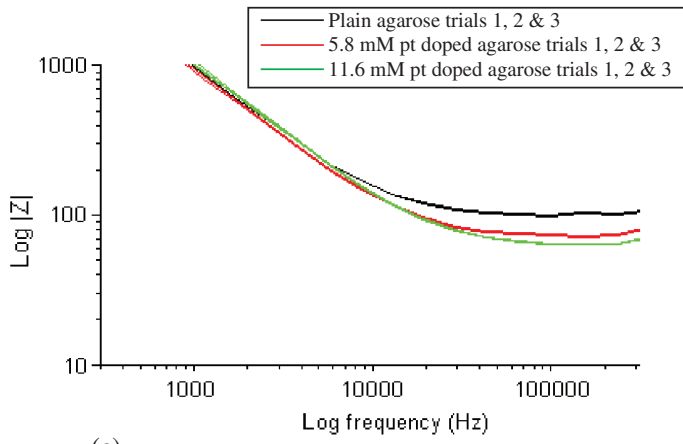

(c)

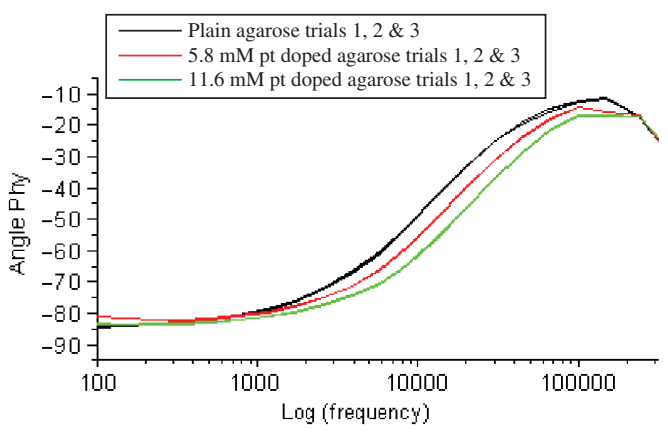

(d)

Fig. 2. (a) Picture of the impedance measurement setup (electrode spacing $=23 \mu \mathrm{m}$, width $=17 \mu \mathrm{m}$ ), (b) Circuit model for analysis (c) Impedance magnitude versus frequency (d) Impedance phase versus frequency.

A PDMS (polydimethyl siloxane) well was mounted for containing and correctly positioning the gel melt over the set of electrodes (see Fig. 2(a)). After the gel solidified, the impedance and phase angle measurements were performed and plotted on a logarithmic scale (see Figs. 2(c) and (d)). A normalization of the system was done by measuring the impedance of deionized water between any two experiments for baseline correction purposes. A simple circuit model for the pair of electrodes immersed in an electrolytic solution is shown in Figure $2(\mathrm{~b})^{21,22}$ where $C_{\mathrm{di}}$ is the dielectric capacitance (it contains dielectric contributions from all materials surrounding the electrodes, including the gel), $R_{\mathrm{s}}$ is the bulk solution resistance (charge transport across the bulk gel), and $Z_{\mathrm{w}}$ is the interfacial impedance, which accounts for the changes in the ionic gradient at the interface and depends mostly on properties of the electrolytes and electrodes. $R_{\text {ser }}$ is the series resistance due to the physical shape of the electrodes, the interelectrode spacing, etc. Each circuit component represents either a physical structure or a factor that affects the measurement. The circuit is modeled mathematically wherein extractions of parameters start with a first estimate of each parameter in the model. ${ }^{23}$ By iterating each of these parameters the predicted and experimental data points were fitted while minimizing the least square error to a tolerance value of $10^{-13}$. When this error values were reached the fitting was terminated and the final values of the parameters were extracted and recorded. Table S1 (supporting information) represents the extracted parameters, with their averages and standard deviations for each trial.

A thin film of the doped gel was prepared by spincoating the molten gel onto a plain silicon substrate, which was then heated in a vacuum oven (at $60{ }^{\circ} \mathrm{C}$ overnight). The silicon substrate with the dried composite film was characterized using a S-4700 Hitachi scanning electron microscope (SEM). Six micro-liters of molten agarose platinum gel were also dispensed on a carbon coated copper grid. A filter paper was used to wick off the excess material after which the grid was stirred in boiling water to make a thin film of the gel and was investigated by transmission electron microscope (JEOL 1200 EX). The gel material was compared for resolution of a $1 \mathrm{Kbp}$ ladder and the resolution was not altered with the new platinum nano-particle doped gel. The comparison of both fractionations was performed using imageJ software. Figure 7(a) shows a comparison of the images and 7(b) shows the band size analysis. The gel image shown in Figure 7(a) has a plain agarose sample in lane 1 and a nanoparticle doped agarose sample in lane 2 highlighted by the selection windows 1 and 2 respectively. Hybrid glass PDMS devices were fabricated with micro-capillaries and filled with agarose and the newly doped agarose samples and DNA samples were translated through these capillaries using electrodes. 


\section{RESULT AND DISCUSSION}

Platinum nano-particles for doping the agarose gels were prepared as hydrosols of two different platinum concentrations by sodium boro-hydride reduction of potassium tetrachloroplatinate with mercapto-succinic acid as a surface protecting group. ${ }^{17,24} \mathrm{UV}$-Vis absorbance spectra of the platinum hydrosols (see supporting information, Fig. S1) resulted in a sharp peak in the $216 \mathrm{~nm}$ region consistent with the presence of platinum nano-particles. ${ }^{20,25}$ TEM showed the formation of spherical platinum nano-particles with a mean diameter of $13 \pm 4 \mathrm{~nm}$ (see supporting information, Fig. S2). Platinum-doped 2\% agarose gels were prepared by heating agarose powder in a 1:4 volumetric ratio mixture of $1 \mathrm{X}$ TAE buffer and platinum hydrosol. Thin films of the doped gel were spun-coated onto glass substrates and examined by field emission scanning electron microscopy (FESEM). A well-distributed array of platinum aggregates (200-250 nm) was observed on the surface of the agarose matrix (see Fig. 3(a)). Electron dispersive spectroscopy (EDS) confirmed the presence of platinum metal in one of the aggregates (see supporting information, Fig. S3). TEM micrographs of a thin section of gel showed a range of platinum particle sizes from the large aggregates observed in the SEM down to approximately $10 \mathrm{~nm}$ particles distributed throughout the matrix (see Fig. 3(b)).
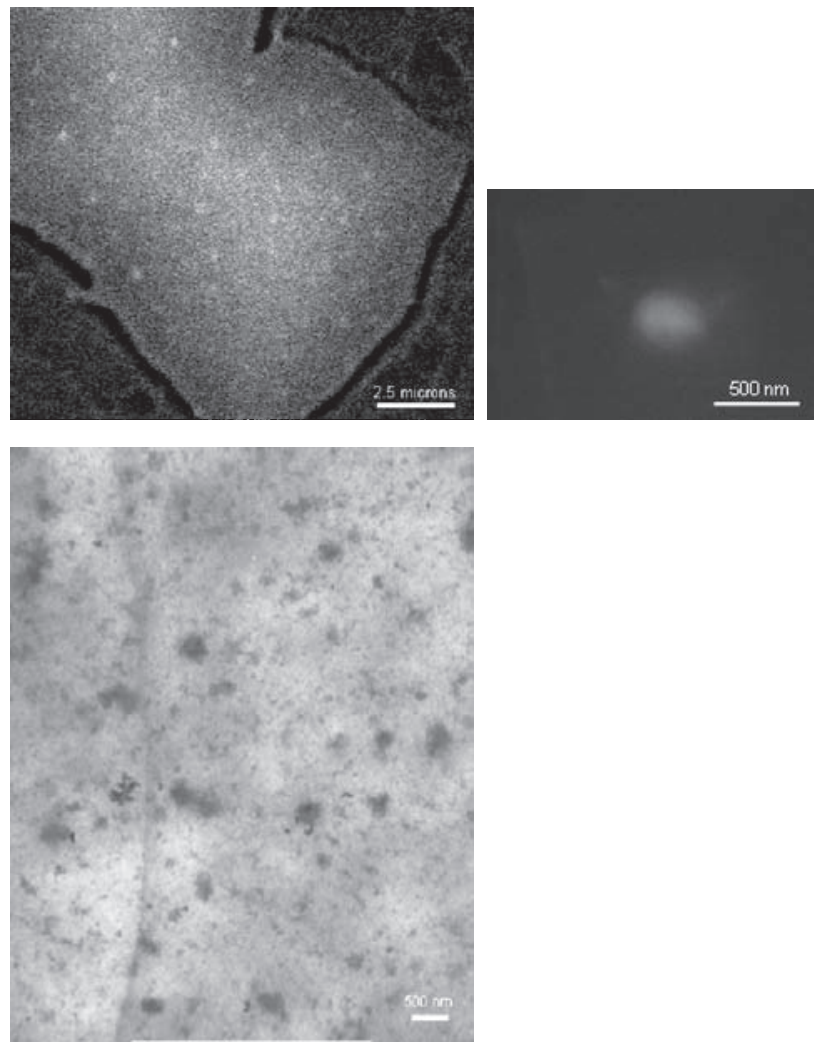

Fig. 3. (a) Array of platinum nano-particles at $10 \mathrm{~K}$ in agarose matrix and snapshot of a single particle at $60 \mathrm{~K}$ magnification. (b) TEM image of an agarose membrane doped with platinum nano-particles.
DNA segments were size-fractionated on doped and undoped gels at 200, 150, 100 and 50 volts. An enhancement in the segment mobility was observed on the platinum nano-particle doped gels. For example, Figure 1 shows a time sequence for the electrophoresis of a $527 \mathrm{bp}$ segment on plain agarose and platinum doped agarose (higher concentration hydrosol) gels at $200 \mathrm{~V}$. A more rapid migration is observed on the doped gel. DNA segment mobility values, as calculated using the 1-D mobility equation $\mu=v / E$ where, $\mu=$ mobility of the stain, $v=$ velocity $(\mathrm{cm} / \mathrm{sec}), E=$ electric field $(\mathrm{V} / \mathrm{cm})$, are plotted with the electric field in Figure 4 for both the doped and undoped gels. ${ }^{22} \mathrm{~A}$ increase in the mobility of the strand with electric field was observed for the doped gel resulting in a mobility increase from $6.6 \times 10^{-5} \mathrm{~cm}^{2} / \mathrm{V} \cdot \mathrm{sec}$ to $9.3 \times 10^{-5} \mathrm{~cm}^{2} / \mathrm{V} \cdot \mathrm{sec}(1.5$ times $)($ Electric Field $=8 \mathrm{~V} / \mathrm{cm})$ from undoped to doped gel. At fields below this, the DNA strand mobility on the doped and undoped gels is not significantly different. The increased mobility does not affect the resolving power of the gel. This is shown by electrophoresis of a 100-1000 bp gene marker on the doped gel (see supporting information, Fig. S4), which showed no change in resolution from the undoped gel.

To understand the cause of the mobility increase, we investigated the electrical properties of the doped and undoped gels. Current-voltage $(I-V)$ measurements indicated an average bulk conductivity of the undoped agarose film of $3.15 \mathrm{~S} \mathrm{~cm}^{-1}$, which changed to $7.2 \mathrm{~S} \mathrm{~cm}^{-1}$ and $14.07 \pm 1.24 \mathrm{~S} \mathrm{~cm}^{-1}$ respectively, on doping with the low and high concentration platinum hydrosols. A plot of the conductivity against the platinum concentration shows an approximately linear relationship (see Fig. 5). We performed impedance spectroscopy on the different gel materials and found a decrease of the characteristic impedance in the range of $100 \mathrm{~Hz}-100 \mathrm{kHz}$ (see Fig. 2(b)). The corresponding range, where the bulk solution resistance dominates (and where it was lower) was also shifted to higher

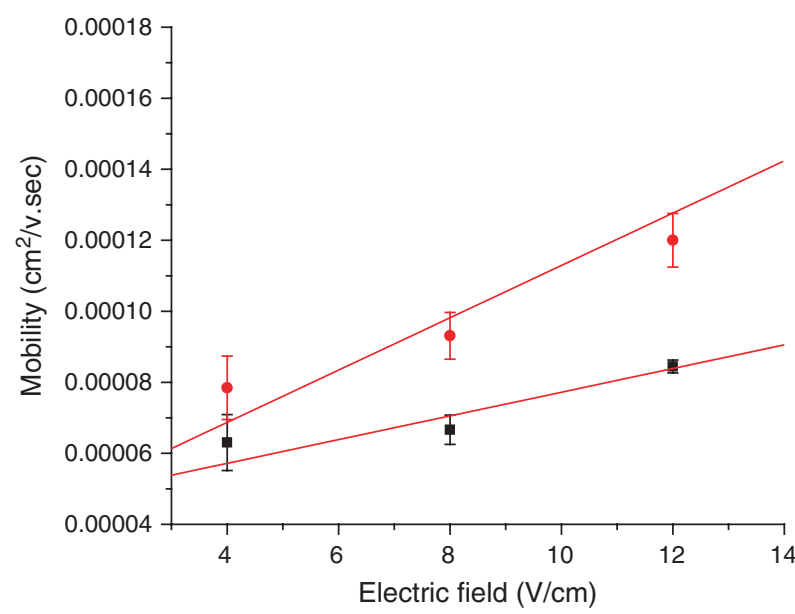

Fig. 4. Mobility plots for plain and doped agarose. (closed red circlesplatinum doped agarose, closedblack squares-plain agarose). 


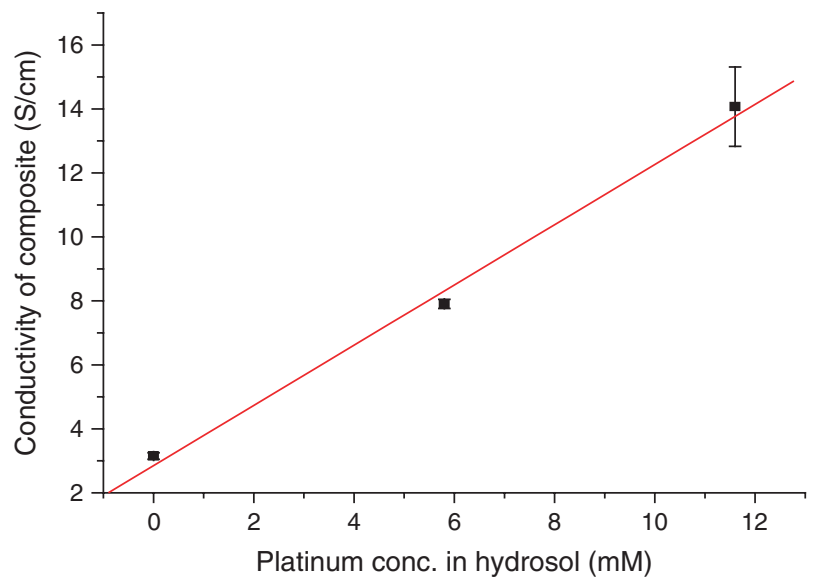

Fig. 5. Plot between gel conductivity $\left(\mathrm{S} \mathrm{cm}^{-1}\right)$ and platinum concentration in the hydrosol $(\mathrm{mM})$ used to prepare the gel.

frequency which can be attributed to an increase in ionic concentration. ${ }^{23}$ Table I in supporting information provides a summary of the average of $R_{\mathrm{s}}$ and $C_{\mathrm{di}}$ and Figure 6 summarizes the extracted parameters $R_{\mathrm{s}}, C_{\mathrm{di}}$ and $R_{\mathrm{ser}}$. It can be noted that the solution resistance goes down from $97.2 \pm 0.8 \mathrm{ohms}$ to about $60.8 \pm 0.6 \mathrm{ohms}$, which is a $37 \%$ decrease. This increased conductivity of the doped gels could arise from the $\mathrm{NaCl}$ and $\mathrm{KCl}$ salts that are co-products in the platinum hydrosols synthesis (increased conductivity due to increased ionic strength).${ }^{17}$ However, as reported earlier by Stellwagen and Stellwagen, this should have resulted in reduced DNA mobility due to the screening of DNA molecules by an increase in counterions in the surrounding ion atmosphere. ${ }^{27}$ Increased electrophoretic mobility in the platinum doped gels can be explained by the dielectric constant enhancement as per the equation, $u=\varepsilon \varepsilon_{0} \zeta / \eta$ where, $u=$ mobility of the ion, $\varepsilon$ is the dielectric constant of the medium, $\zeta$ is the zetapotential of the ion and $\eta$ is the viscosity of the medium. ${ }^{28}$ The viscosity of the medium typically depends on the percent agarose in the gel. This remains unaltered in our case in both the doped and undoped gels. As DNA is highly negatively charged it does not have an alteration in its zeta potential value because of a change in the surrounding ion atmosphere. ${ }^{22}$ Thus, the increase in the mobility must arise from an enhancement of the dielectric capacitance indicating an increase in the dielectric constant of the medium due to the presence of the dispersed platinum nano-particles. The data in figure supports this theory. The dielectric capacitance increased from $1.77 \pm 0.12 \mathrm{nF}$ to $2.37 \pm 0.02 \mathrm{nF}$ which is an increase of 1.34 times when the platinum concentration is increased from 0 to $11.6 \mathrm{mM}$. Naka et al..$^{26}$ have reported an increase in dielectric constant of poly(vinyl chloride) using poly (2-methyl-2-oxazoline) coated gold nanoparticles which are made with a similar reduction chemistry. Simultaneously an increased conductivity of the gel material obviously indicates an increase in current and joule heating.
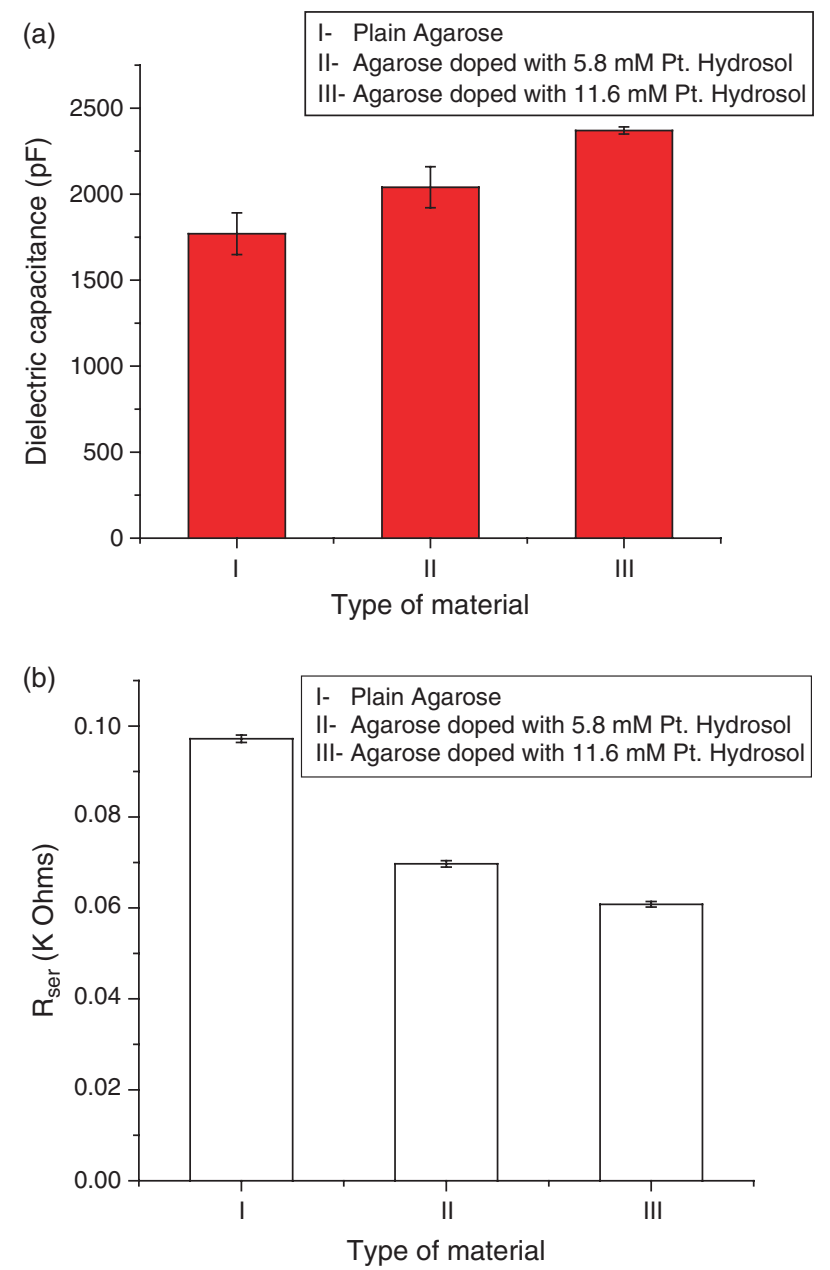

Fig. 6. Comparison of gel (a) dielectric capacitance and (b) resistance of the agarose and platinum doped agarose (5.8 and $11.6 \mathrm{mM})$.

However substantial Joule heating would have changed the resolution of the gel material by band broadening due to temperature gradients set within the gels. ${ }^{18}$ Band broadening effects become prominent with temperature increase and temperature gradients setup within the gels during electrophoresis and mechanistically happens due to lateral diffusion of the DNA as it meets varying pore sizes while translating one dimensionally within the gel material. Figures 7(a) and (b) show a band width comparison between both plain agarose and nanoplatinum doped agarose samples. The overall areas occupied by the bands in lanes 1 and 2 are 14.25 AU (arbitrary units) and 13.8 AU respectively. The peaks in this analysis signify each measured peak with its size as a percent of the total size of the measured peaks and they show an average error of less than 0.0064, which is an order of magnitude lower than the measured peak values. Some difference is observed in the $500 \mathrm{bp}$ band. The peak area varies from $10 \%$ of the total size of the measured peak in the plain agarose gel to $17 \%$ of the total size of the measured peaks in the nanoplatinum doped gel samples. This is mainly because of the 


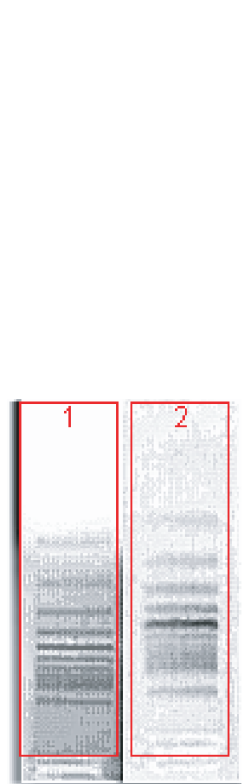

(a)

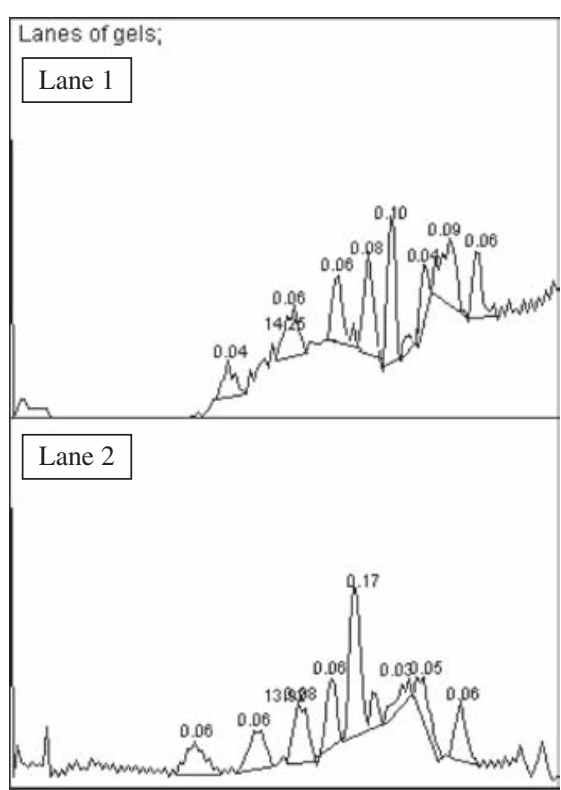

(b)
Fig. 7. ImageJ analysis for studying band broadening effects between (a) images of both lanes (1) Plain agarose and (2) Nanoparticle doped agarose. (b) Analysis for comparison of band sizes in both cases. (no appreciable broadening observed).

fact that the band in the nanoplatinum doped gel material (Fig. 7(b), Lane 2) seems to have a kink in the right hand lower corner. We believe that this just due to a localized formulation problem of the gel material at this spot. Had it been a continuous phenomena then all bands would have this kink. Thus the nanoparticle doped agarose gel samples do not exhibit any band broadening effects indicating insignificant joule heating due to the nanoparticles and associated conductivity increase.

Thus, our work demonstrates the use of novel nanoparticle doped gel formulations to increase the DNA mobility and correspondingly reduce the time of running the gel. The fact that the resistance can be lowered can also allow us to use a lower voltage for containing the same separation resolution as is possible with conventional gel formulations.

\section{CONCLUSIONS}

We have developed a new platinum agarose composite material with enhanced sample mobility and increased conductivity. The sample mobility in the composite increases from $6.6 \times 10^{-5} \mathrm{~cm}^{2} / \mathrm{V} \cdot \mathrm{sec}$ to $9.3 \times 10^{-5} \mathrm{~cm}^{2} / \mathrm{V}$. $\sec (1.5$ fold $)$ at low $(8 \mathrm{~V} / \mathrm{cm})$ electric field values. The slope of the mobility versus electric field increased by two-fold. The DC conductivity of the new composite was found to increase five-fold. We have further performed impedance spectroscopy on the gel samples using inter-digitated electrodes and found a substantial reduction in the medium impedance in frequency range
$100 \mathrm{~Hz}-100 \mathrm{kHz}$. We have modeled the impedance data with a simple parallel RC circuit and used an iterative program to extract the dielectric capacitance and gel resistance information. We found a 1.34 times increase in the gel dielectric capacitance and a 37\% reduction in gel resistance with increasing platinum concentration. We believe that the observed mobility increase is attributed to an increased conductivity and enhancement of dielectric constant of the medium. Application of this novel gel material to low voltage capillary electrophoresis in micro-channels has been demonstrated and reported separately. The new gel material does not show any substantial temperature increase and leads to insignificant band broadening effects.

\section{SUPPORTING INFORMATION}

Table S1. Summary of parameters generated from curve fit. (values, averages and standard deviations).

\begin{tabular}{lccc}
\hline Type of material & $\begin{array}{c}R_{\mathrm{s}} \\
(\mathrm{Ohm})\end{array}$ & $\begin{array}{c}C_{\mathrm{di}} \\
(\mathrm{nF})\end{array}$ & $\begin{array}{c}R_{\text {ser }} \\
(\mathrm{Ohm})\end{array}$ \\
\hline Plain agarose & 98 & 1.64 & $1.00 \mathrm{E}-7$ \\
& 96.4 & 1.88 & $1.00 \mathrm{E}-7$ \\
& 97.3 & 1.79 & $1.00 \mathrm{E}-7$ \\
Average & 97.2 & 1.77 & $1.00 \mathrm{E}-7$ \\
Std. dev. & 0.8 & 0.12 & 0 \\
Agarose doped with & 70.4 & 1.9 & $1.00 \mathrm{E}-7$ \\
$\quad 5.8$ mM platinum & 69.7 & 2.09 & $1.00 \mathrm{E}-7$ \\
$\quad$ hydrosol & 69.0 & 2.12 & $1.00 \mathrm{E}-7$ \\
Average & 69.7 & 2.04 & $1.00 \mathrm{E}-7$ \\
Std. dev. & 0.07 & 0.12 & 0 \\
Agarose doped with & 61.5 & 2.39 & $1.00 \mathrm{E}-7$ \\
$\quad 11.6$ mM platinum & 60.4 & 2.35 & $1.00 \mathrm{E}-7$ \\
$\quad$ hydrosol & 60.5 & 2.38 & $1.00 \mathrm{E}-7$ \\
Average & 60.8 & 2.37 & $1.00 \mathrm{E}-7$ \\
Std. dev. & 0.061 & 0.21 & 0 \\
\hline
\end{tabular}

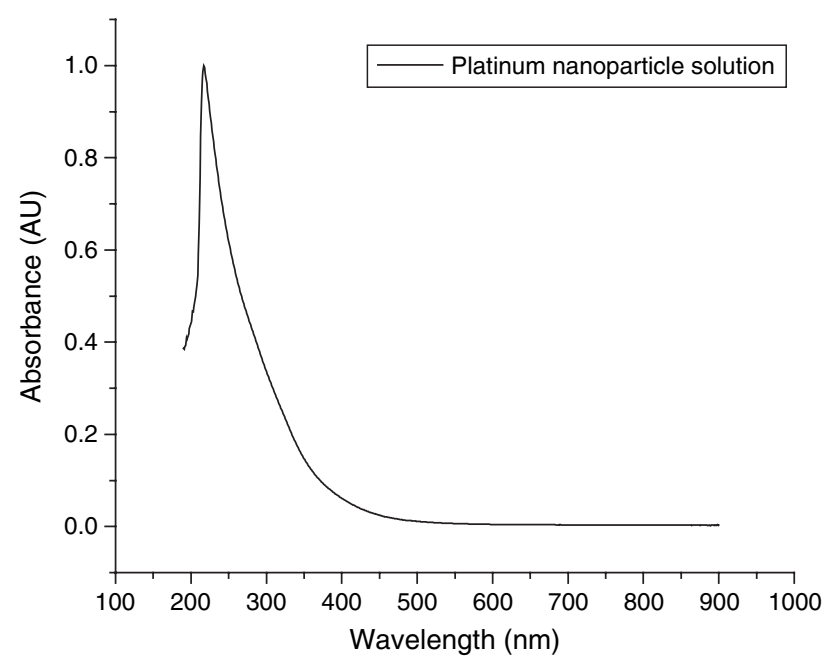

Fig. S1. UV-Vis absorbance spectra of Platinum hydrosol. 
(a)

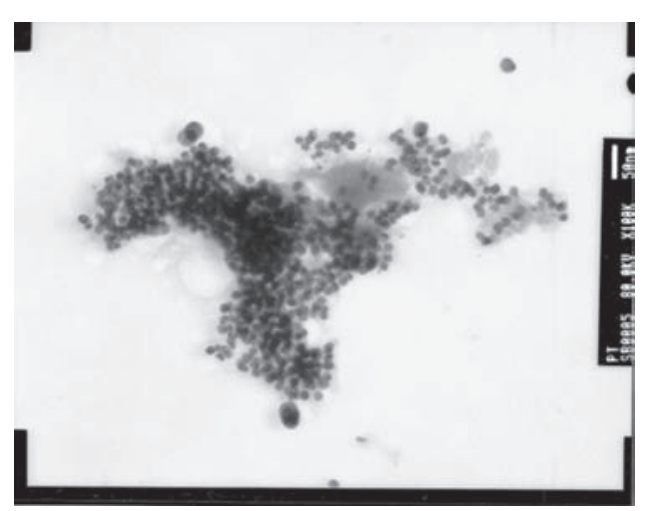

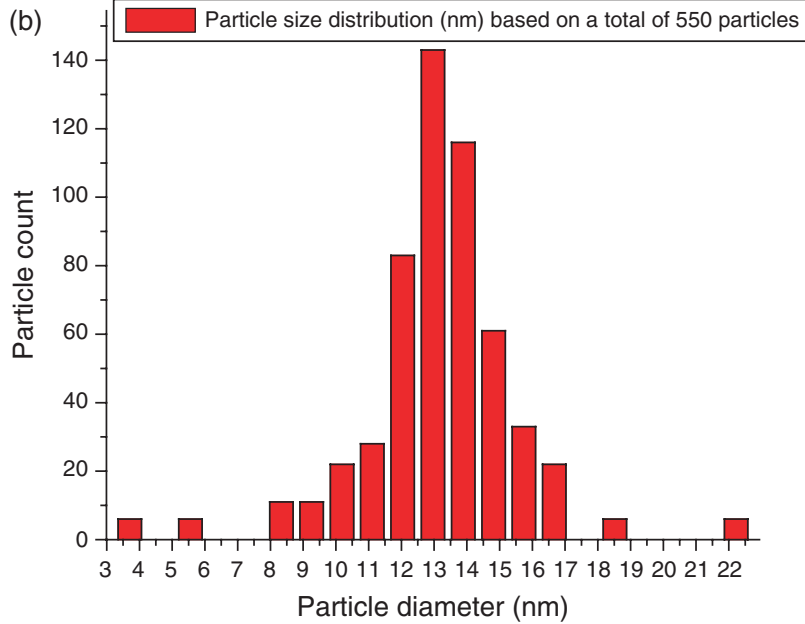

Fig. S2. (a) TEM image of Platinum nano-particles (b) Particle size distribution.

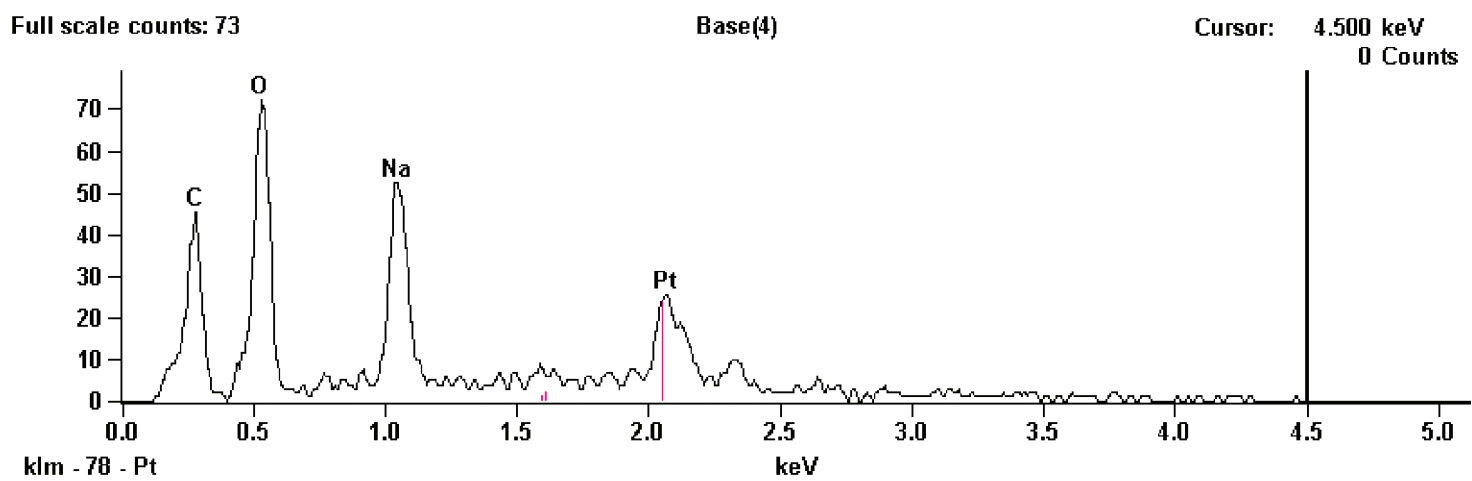

Fig. S3. EDS spectrum of the platinum nano-particle taken at $30 \mathrm{KV}$ accelerating voltage. (No PtCl peak at 2.62 indicating full reduction of chloroplatinate salt).

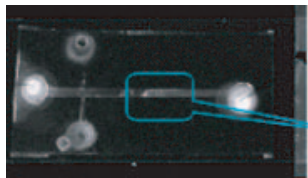

(a)
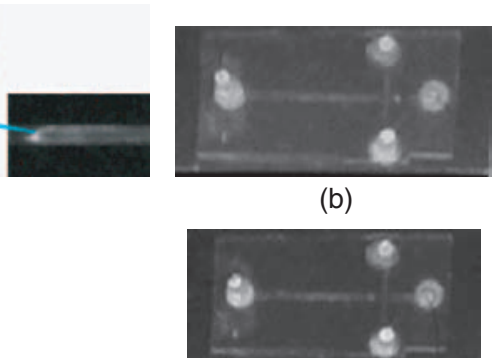

(c)

Fig. S4. Capillary electrophoresis of a 750 bp nucleic acid segment in (a) in pure agarose $(300 \mathrm{~V})$, (b) in agarose with filler nano-particles. $(65 \mathrm{~V}$, Electric field $=25 \mathrm{~V} / \mathrm{cm})(\mathrm{c})$ in agarose with filler nanoparticles. $(65 \mathrm{~V}$, Electric field $=25 \mathrm{~V} / \mathrm{cm})$ with electrode polarity reversed. Reprinted with permission from [19], S. Bhattacharya et al., Sens. Letts. (2008). () 2008, accepted for publication.

Acknowledgment: The authors gratefully acknowledge the financial support from the National Pork Board and the National Institute of Health. They also gratefully acknowledge the usage of the Birck Nanotechnology Center facilities at Purdue University for conducting impedance spectroscopy.

\section{References and Notes}

1. A. S. Cohen, D. R. Najarian, A. Paulus, A. Guttman, J. A. Smith, and B. L. Karger, Proc. Natl. Acad. Sci. USA 85, 9660 (1988).

2. A. Manz and H. Minhas, Lab on Chip 2, 1N (2002).

3. A. Manz and J. C. T. Eijkel, P. Appl. Chem. 73, 1555 (2001).

4. D. R. Reyes, D. Iossifidis, P. A. Auroux, and A. Manz, Anal. Chem. 74, 2623 (2002).

5. P. Camilleri, Capillary Electrophoresis, Theory and Practice, CRC press Inc., Florida (2000).

6. M. N. Albarghouthi, B. A. Buchholz, E. A. S. Doherty, F. M. Bogdan, H. Zhou, and A. E. Barron, Electrophoresis 22, 737 (2001).

7. A. E. Barron, W. M. Sunada, and H. W. Blanch, Biotechnol. Bioeng. 52, 259 (1996)

8. L. M. Fu and R. J. Yang, Electrophoresis 24, 1253 (2003).

9. J. Lu and C. P. Wong, Electronic Components and Technology Conference (IEEE) 1033 (2007).

10. J. Xu and C. P. Wong, Electronic Components and Technology Conference (IEEE) 2031 (2004).

11. D. L. Ma, R. W. Seigel, J. I. Hong, L. S. Schadler, E. Martensson, and C. Onneby, J. Mater. Res. 19, 857 (2004).

12. D. L. Ma, T. A. Huegner, R. W. Seigel, E. Christerson, E. Martensson, C. Onneby, and L. S. Schadler, Nanotechnology 724 (2005).

13. M. Muszynska, H. Wycislik, and M. Siekierski, Solid State Ionics 147, 281 (2002). 
14. B. Oh, W. Jung, D. W. Kim, and H. W. Rhee, Bull. Korean Chem. Soc. 23, 683 (2002).

15. M. Morita, H. Noborio, N. Yoshimoto, and M. Ishikawa, Solid State Ionics 177, 715 (2006).

16. W. Wieczorek, Z. Florjanczyk, and J. R. Stevens, Electrochim. Acta 40, 2251 (1995)

17. S. Chen and K. J. Kimura, Phys. Chem. B 105, 5397 (2001).

18. J. A. Luckey and L. M. Smith, Anal. Chem. 65, 2841 (1993).

19. S. Bhattacharya, D. Gangopadhyay, N. Chanda, Y. Liu, S. A. Grant, P. R. Sharp, R. Bashir, K. Gangopadhyay, and S. Gangopadhyay, Sensor Letters (2008), accepted for publication.

20. H. H. Ingelsten, R. Bagwe, A. Palmqvist, M. Skoglundh, C. Svanberg, K. Holmberg, and D. O. Shah, J. Colloid Interface Sci. 241, 104 (2001).

21. P. Jacobs, W. Hofer, R. Rossau, A. V. D. Voorde, P. V. Gerwen, and P. Detemple, Proceeedings of the Second International Conference on Microreaction Technology, New Orleans, LA, USA (1998), p. 223.

22. D. M. Hawcroft, Electrophoresis: The Basics, Oxford University Press, New York (1997).

23. R. Gomez, R. Bashir, and A. K. Bhunia, Sens. Actuators, B 86, 198 (2002).

24. S. Y. Zhao, S. H. Chen, S. Y. Wang, D. G. Li, and H. Y. Ma, Langmuir 18, 3315 (2002).

25. J. F. Rivadulla, M. C. Vergara, M. C. Blanco, M. A. Lopez-Quintela, and J. Rivas, J. Phys. Chem. B 101, 8997 (1997).

26. K. Naka, H. Itoh, S. Y. Park, and Y. Chujo, Polym. Bull. 52, 171 (2004).

27. E. Stellwagen and N. C. Stellwagen, Electrophoresis 23, 1935 (2002).

28. A. J. Bard and L. R. Faulkner, Electrochemical Methods, Wiley, New York (1980).

Received: 2 October 2008. Accepted: 10 November 2008. 